\title{
A Rare Case of Perimesencephalic Subarachnoid Hemorrhage in a Postpartum Patient with Preeclampsia
}

\author{
Jessica C Morgan ${ }^{1 *}$ and Nausheen Zaidi ${ }^{2}$ \\ ${ }^{1}$ Department of Obstetrics and Gynecology, University of Illinois at Chicago, USA \\ ${ }^{2}$ Department of Maternal Fetal Medicine, Advocate Healthcare-Christ Hospital, USA
}

\section{INTRODUCTION}

Perimesencephalic subarachnoid hemorrhage (SAH) is a subset of subarachnoid hemorrhage not associated with an aneurysm. Most cases of non-traumatic SAH are caused by a ruptured intracranial aneurysm, although about $15-20 \%$ of patients will have no identifiable lesion on angiography [1]. Perimesencephalic $\mathrm{SAH}$ is further differentiated from non-aneurysmal SAH due to its localized pattern of bleeding and benign course. The incidence of perimesencephalic SAH is estimated at 0.5 per 100,000 people per year, and unlike aneurysmal SAH, it has not been shown to have higher prevalence in women. Women account for $42 \%$ of perimesencephalic SAH cases [2]. SAH in pregnancy is a rare, but devastating event. One study from 2016 showed a prevalence of SAH in 5.8 per 100,000 deliveries, with $66.7 \%$ of SAH occurring in the postpartum period. It has also been shown that the prevalence of peripartum SAH is increasing drastically, having been less than 4 per 100,000 admissions in 1995. This increase has been shown after controlling for race, age and hypertensive disorders [3]. In light of this increased risk of such a devastating complication of pregnancy, it is important to understand the signs and symptoms of SAH regardless of a patient's risk factors. Our case report describes a patient with no prior history of hypertension who developed preeclampsia in the post-partum period, and subsequently suffered a perimesencephalic SAH. To our knowledge, this is the first reported case of perimesencephalic subarachnoid hemorrhage in both the post-partum period and with co-existing pre-eclampsia.

\section{CASE REPORT}

Our patient is a 31-year-old gravida-four-para-three-zero-zerothree who presented at thirty-seven weeks gestation with abdominal pain at the site of her prior cesarean scar. Due to her history of three cesarean sections, uterine contractions and incisional pain, the decision was made to proceed with a repeat cesarean section that day. Cesarean section and bilateral tubal sterilization were uncomplicated with an estimated blood loss of $700 \mathrm{~mL}$. She was discharged home on post-operative day two after an uncomplicated recovery. She then presented to obstetric triage on post-operative day four with headache and blurred vision. She was diagnosed with pre-eclampsia with severe features by transaminitis with a peak aspartate transferase of 160 and peak alanine transferase of 109 . Her blood pressures were elevated in the mild range but never the severe range and she did not require any antihypertensive therapy. She was managed with magnesium sulfate for twenty-four hours and was discharged home on post-operative day six.

On post-operative day seven, she presented again to obstetric triage with a new-onset severe headache and photophobia. She had persistently elevated blood pressures in the severe range at this time and was given nifedipine immediate release due to lack of intravenous access. This appropriately controlled her blood pressure and she required no further medication. Due to the severe nature of her headache she underwent non-contrast head computed tomography (CT) scan. The scan revealed an acute subarachnoid hemorrhage centered within the basilar cisterns. Neurology and neurosurgery were urgently consulted to evaluate the patient who then underwent CT angiography with and without contrast of the head and neck. These studies revealed unchanged subarachnoid hemorrhage in the basilar cisterns extending to the perimesencephalic, suprasellar, prepontine, and right cerebellopontine angle cisterns with no obvious cerebral aneurysm,
Quick Response Code:

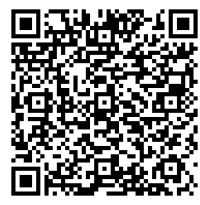

Address for correspondence: Jessica C Morgan, Department of Obstetrics and Gynecology, University of Illinois at Chicago, USA

Received: March 20, 2020 Published: March 31, 2020

How to cite this article: Jessica CM, Nausheen Z. A Rare Case of Perimesencephalic Subarachnoid Hemorrhage in a Postpartum Patient with Preeclampsia. 2020 - 2(2) OAJBS. ID.000158. DOI: $10.38125 / \mathrm{OAJBS} .000158$ 
arteriovenous malformation, vasospasm or other vascular lesion to explain the subarachnoid hemorrhage.

The patient was admitted to the neurological critical care unit and treated with nimodipine 60 milligrams every four hours and goal systolic blood pressure less than 140 millimeters of mercury. The patient ultimately did not require any additional antihypertensive medication and showed adequate blood pressure control for the remainder of hospital admission. The patient underwent an echocardiogram which was within normal limits, and the following day a cerebral angiogram was performed. The angiogram was negative for any aneurysm, arteriovenous malformation (AVM) or dural arteriovenous fistula (AVF). The diagnosis remained perimesencephalic bleed at this time.

Per protocol, the diagnostic cerebral angiogram was repeated after one week and still showed no aneurysm, AVM or AVF. The patient remained neurologically intact with no focal or generalized deficits throughout the entire admission. Her headaches subsided and nimodipine was stopped after confirming that there was no aneurysm or evidence of vasospasm. She was discharged home in stable condition on hospital day eight, post-operative day fifteen.

\section{DISCUSSION}

There are multiple theories about the cause of bleeding in perimesencephalic SAH including rupture of a perforating artery, venous origin of bleeding and basilar artery wall hematoma. A venous source of bleeding has been suggested due to the lack of extensive blood flow and low rate of rebleeding seen in perimesencephalic SAH. It has been observed that many cases occur after physical exertion, which causes increased intrathoracic pressure, impaired jugular venous return, elevated intracranial venous pressure and leakage of venous blood from these vessels [4]. Although the pathophysiology may be different than the typical aneurysmal SAH, the clinical presentation is often similar. Patients will typically present with sudden onset headache, nausea and vomiting, though it may be less severe than patients with an aneurysm [2].

Perimesencephalic SAH is defined by bleeding confined to the cisterns around the midbrain and is typically associated with a benign course and low risk for rebleeding [6]. It has been described during pregnancy twice, but never in the postpartum period. In these two prior case reports, both patients presented during pregnancy, one in the first trimester and one in the third, with acute onset of headache and neck pain. They both underwent CT scans showing acute subarachnoid hemorrhage and CT angiogram without evidence of an aneurysm. Both patients were ultimately discharged home with no further neurologic issues [6]. Criteria for diagnosing perimesencephalic SAH include hemorrhage located anterior to the midbrain without extension to the anterior part of the ambient cistern or basal part of the Sylvian fissure, incomplete filling of the anterior interhemispheric fissure, no extension to the lateral Sylvian fissure, and absence of frank intraventricular hemorrhage [7]. Perimesecenphalic SAH occurs in the absence of aneurysmal disease. While it is known that the changes of cerebral hemodynamics during pregnancy may cause increased cerebral blood flow, which can be expected to aggravate an existing lesion such as an aneurysm, there is no application of this theory to nonaneurysmal hemorrhages [8].

Furthermore, this entity has not been previously described in a patient with pre-eclampsia, which our patient did meet criteria for based on severely elevated blood pressures and transaminitis.
The mechanism by which pre-eclampsia can cause intracranial hemorrhage has been suggested. In settings of new acute hypertension, the arterial pressure rises above the cerebral blood flow autoregulation range, and this causes increased intravascular pressures. The result is an increased pressure on arteries and arterioles which can lose their ability to provide vascular resistance, therefore causing endothelial damage, edema and risk of brain injury. The damage to the blood brain barrier can cause acute intracranial hemorrhage, which remains exceedingly rare [9]. It has been suggested that intracerebral hemorrhage is found in up to $40 \%$ of patients with eclampsia during autopsy [10]. So, while it is accepted that severe hypertension and hypertensive disorders of pregnancy may increase the risk of intracranial hemorrhage, it does not quite explain the phenomenon of perimesencephalic $\mathrm{SAH}$ which is often not arterial.

Another point of interest when discussing SAH in the obstetric patient, is the use of spinal anesthesia. Spinal anesthesia involves a dural puncture in order to enter the subarachnoid space, though complications such as dural puncture headache are not always noted. Numerous case reports have reported not only post-dural puncture headaches, but subarachnoid hemorrhage because of spinal anesthesia. One such case report involved a post-partum patient receiving spinal anesthesia for removal of retained placenta. Shortly after uncomplicated induction of spinal anesthetic, the patient experienced an excrutiating headache and was subsequently diagnosed with a SAH, also without any apparent lesion on angiogram. Repeat angiogram also confirmed no obvious lesion and the patient made a full recovery. It is theorized that low cerebrospinal fluid (CSF) pressure can be caused by the dural puncture even without development of a significant dural puncture headache. This can then cause a severe decrease in intracranial pressure, thereby causing an increase in pressure across the arterial wall which ultimately ruptures a previous vascular malformation [11]. Again, this theory relates to arterial malformations and arterial sources of SAH, but it is pertinent for the discussion of obstetric patients as they commonly receive spinal anesthesia. Our patient did indeed receive spinal anesthesia for her cesarean section and did not present with a severe headache until POD\#6 after already being diagnosed with pre-eclampsia.

\section{CONCLUSION}

The risks of intracranial hemorrhage in the obstetric patient are rare but can be life threatening if unrecognized and untreated. It is important to remember the common risk factors that many obstetric patients carry, including hypertensive disease, maternal efforts of labor such as Valsalva during pushing that may exacerbate previously undiagnosed vascular lesions, and the risks of anesthesia often used during the labor and delivery process. While SAH is exceedingly rare, and perimesencephalic SAH even moreso, it is important to be appropriately concerned when an obstetric or postpartum patient presents with headache, neck pain, or photophobia which are common presenting symptoms of these catastrophic complications.

\section{REFERENCES}

1. Schwartz TH, Solomon RA (1996) Perimesencephalic nonaneurysmal subarachnoid hemorrhage: review of the literature. Neurosurgery 39(3): 433-440.

2. Mensing LA, Vergouwen MDI, Laban KG (2018) Perimesencephalic Hemorrhage: A Review of Epidemiology, Risk Factors, Presumed Cause, Clinical Course, and Outcome. Stroke 49(6): 1363-1370. 
3. Bateman BT, Olbrecht VA, Berman MF (2012) Peripartum subarachnoid hemorrhage: nationwide data and institutional experience. Anesthesiology 116(2): 324-333.

4. Matsuyama T, Okuchi K, Seki T (2006) Perimesencephalic nonaneurysmal subarachnoid hemorrhage caused by physical exertion. Neurol Med Chir (Tokyo) 46(8): 277-281.

5. Van Gijn J, Van Dongen K, Vermeulen M, Hijdra A (1985) Perimesencephalic hemorrhage a nonaneurysmal and benign form of subarachnoid hemorrhage. Neurology 35(4): 493-497.

6. Hirsch KG, Froehler MT, Huang J, Ziai WC (2009) Occurrence of perimesencephalic subarachnoid hemorrhage during pregnancy. Neurocrit Care 10(3): 339-343.

7. Rinkel GJ, Wijdicks EF (1991) Nonaneurysmal perimesencephalic subarachnoid haemorrhage: CT and MR patterns that differ from aneurysmal rupture. Am J Neuroradiol 12(5): 829-834.

8. Hammer ES, Cipolla MJ (2015) Cerebrovascular Dysfunction in Preeclamptic Pregnancies. Curr Hypertens Rep 17(8): 64.

9. Martin JN, Thigpen BD, Moore RC, Rose CH, Cushman J, et al. (2005) Stroke and severe preeclampsia and eclampsia: a paradigm shift focusing on systolic blood pressure. Obstet Gynecol 105(2): 246-254.

10. Richards A, Graham D, Bullock R (1988) Clinicopathological study of neurological complications due to hypertensive disorders of pregnancy. J Neurol Neurosurg Psychiatry 51(3): 416-421.

11. Eggert SM, Eggers KA (2001) Subarachnoid haemorrhage following spinal anaesthesia in an obstetric patient. Br J Anaesth 86(3): 442-444. 